\title{
Unprecedented uniform 3D growth integration of 10-layer stacked Si nanowires on tightly confined sidewall grooves
}

Ruijin $\mathrm{Hu}^{\dagger}$, Shun $\mathrm{Xu}^{\dagger}$, Junzhuan Wang ${ }^{\dagger *}$, Yi Shi ${ }^{\dagger}$, Jun $\mathrm{Xu}^{\dagger}$, Kunji $\mathrm{Chen}^{\dagger}$ and Linwei $\mathrm{Yu}^{\dagger *}$

${ }^{\dagger}$ National Laboratory of Solid State Microstructures/School of Electronics Science and

Engineering/Collaborative Innovation Centre of Advanced Microstructures, Nanjing University, 210093 Nanjing, P. R. China, Email : wangjz@nju.edu.cn, yulinwei@nju.edu.cn
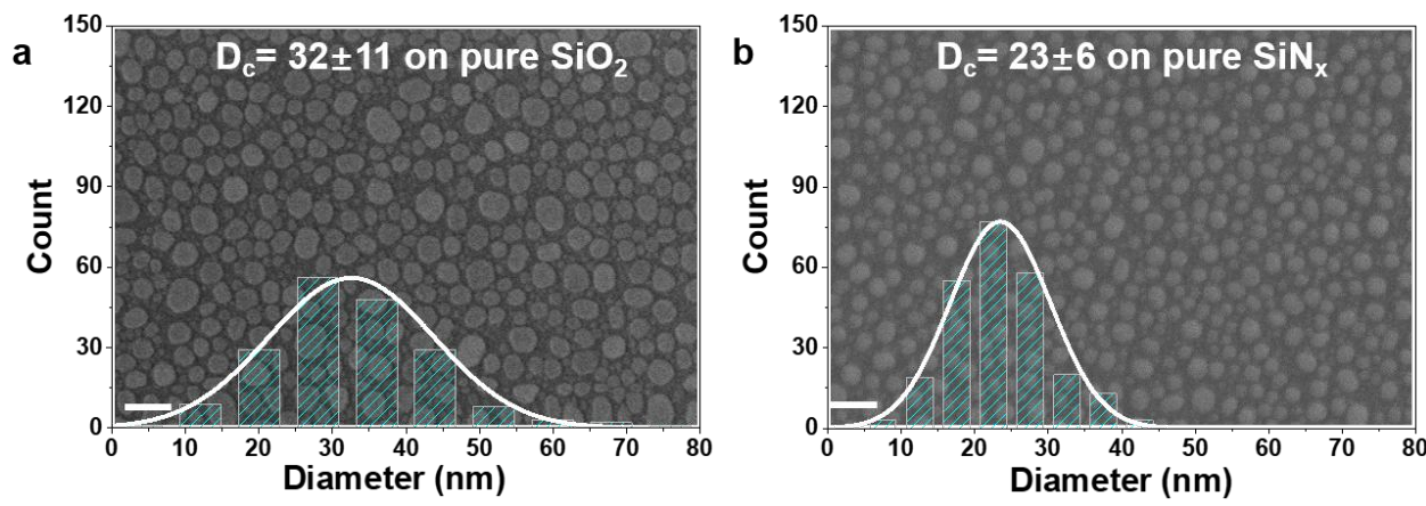

Figure S1. SEM images and catalyst size distribution statistics of the In droplets deposited via EBE evaporation upon the (a) $\mathrm{SiO}_{2}$ and (b) $\mathrm{SiN}_{\mathrm{x}}$ substrates. 

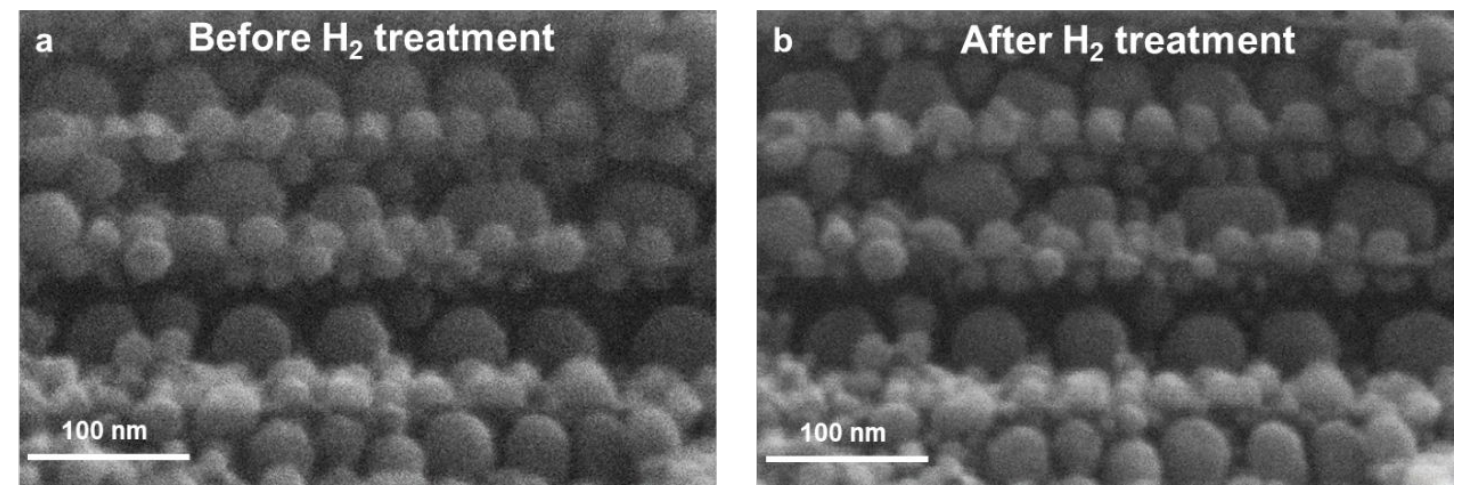

Figure S2. (a) and (b) show SEM images of the In droplets evaporated on the hetero- $\mathrm{SiO}_{2} / \mathrm{SiN}_{\mathrm{x}}$ sidewall grooves before and after $\mathrm{H}_{2}$ plasma treatment, respectively.

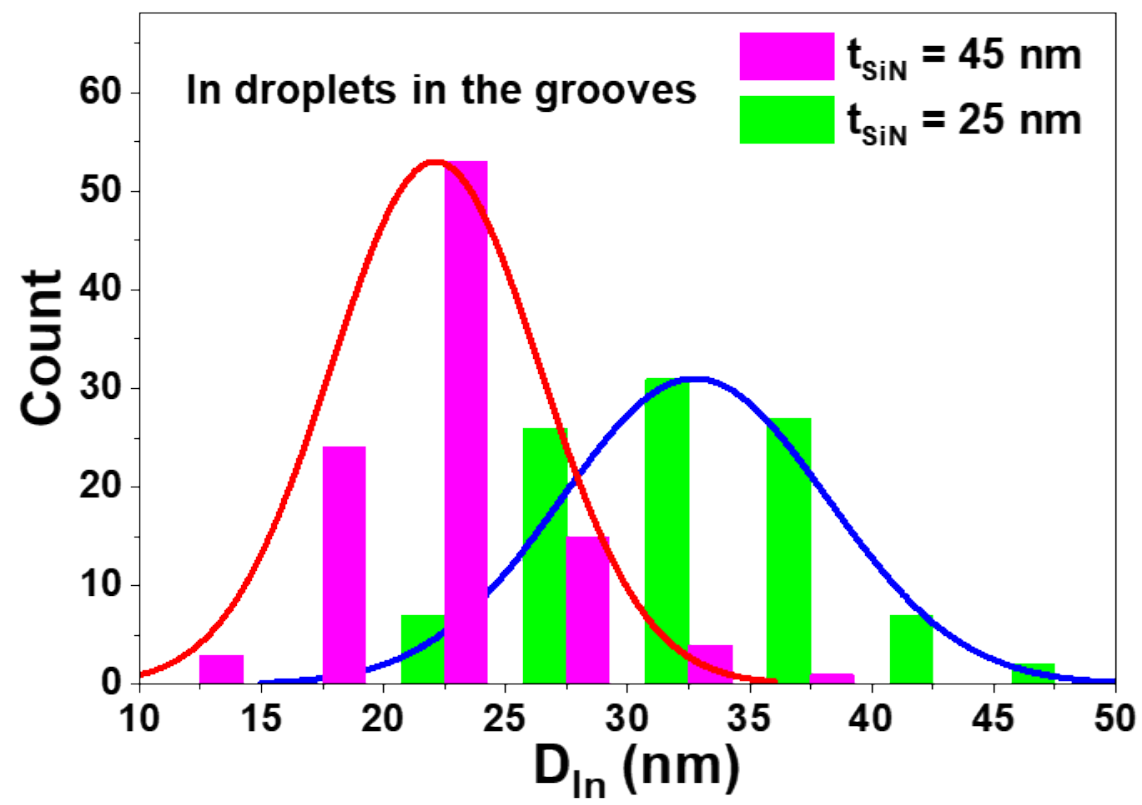

Figure S3. Statistics on the diameter distributions of the In droplets formed in the grooves with different $\mathrm{SiN}_{\mathrm{x}}$ edge thicknesses. 